\title{
MIMO Capacity OF MUltipath-Clustered Channels Using Fourier TRANSFORM TECHNIQUES
}

\author{
Guangze Zhao, Sergey Loyka \\ School of Information Technology and Engineering (SITE) \\ University of Ottawa, 161 Louis Pasteur, Ottawa, Ontario, K1N 6N5, Canada \\ Email: gzhao@site.uottawa.ca, sloyka@site.uottawa.ca
}

\begin{abstract}
Fading correlation is one of the fundamental limits on the performance of multi-antenna (MIMO) systems. Accurate modeling of the angular probability density function (pdf) of the incoming multipath is essential for accurate prediction of the correlation and, hence, the MIMO capacity. Previous studies were limited to the case of single-cluster distribution or uniform pdf. Measurements indicate that multipath often arrives in several angular clusters and with non-uniform angular cluster pdf. We begin with the popular single cluster model and extend it to the case of multi-cluster channel. The single cluster model with various cluster pdfs is studied in both the angular domain and the angular spectrum domain (u-domain). Using Fourier transform techniques, it is shown that the spatial correlation is the inverse Fourier transform of a scaled and shifted version of the cluster pdf in the u-domain. We further prove that the spatial correlations for all the pdfs is roughly the same given the same angular spread. It is shown that the correlation in the two clusters channel has an oscillatory behavior as antenna spacing increases regardless of multipath AOA distributions; the envelope of the correlation is determined by the single cluster angular spread while the oscillations within the envelope are determined by the inter-cluster angular spread. The cluster angular pdf has negligible impact on the MIMO capacity and, hence, simpler uniform model can be used without degradation in accuracy.
\end{abstract}

Index Terms-Wireless propagation channel, fading correlation, Fourier Transform, MIMO capacity

\section{INTRODUCTION}

Understanding of spatial fading correlation is crucial for accurate prediction of multi-antenna (MIMO) system performance. It is well know that the fading correlation in spatially separated antennas depends on the angular distribution of the incoming multipath [1]. While some special cases of the angular pdf have been investigated [18], no detailed understanding of the problem has been achieved so far. Most studies were limited to the case of single cluster with uniform [1-2] or non-uniform pdf [3-5]. Only preliminary studies of multi-cluster channels have been reported [6-7]. It is important to understand the correlation behavior of multi-clustered channels with nonuniform pdf since measurements of practical channels indicated that multipath often arrives in multiple clusters and with non-uniform angular pdf $[5,8]$.

In this paper, we extend Salz-Winters model (single cluster with uniform pdf) to account for more than one cluster and study the effect of clustering with different angular pdfs in details. The emphasis is on analytical analysis, which is further validated using Monte-Carlo simulations. Using the well-known Fourier transform techniques, we demonstrate that the spatial correlation is mainly determined by the angular spread, and the exact shape of angular spectrum is negligible if the angular spread is small. A case of two symmetrical clusters is studied in details, including uniform, triangle, truncated Gaussian, and truncated Laplacian pdfs. Using the developed correlation models and the technique presented in [2], we study the MIMO channel capacity. In particular, we demonstrate that the angular pdf shape has negligible effect on the MIMO channel capacity provided that the distribution parameters (i.e. angular spread and mean AOA) are fixed.

An extension to the case of n-cluster scenario is straightforward. The major analytical techniques developed above are applicable in this general case as well. The results presented agree well with those published in the literature earlier and extend them to the case of multi-cluster channels.

\section{Single-Cluster Channel Model}

In this model, all the multipath components are assumed to arrive to the Rx array within $\pm \Delta / 2$ of the mean angle of arrival (AOA) $\varphi$ (see fig. 1) in the horizontal plane (i.e., this is a 2-D model). The AOA probability density function is assumed to be uniform,

$$
f_{\theta}(\theta)=\left\{\begin{array}{c}
1 / \Delta,-\Delta / 2+\varphi \leq \theta \leq \Delta / 2+\varphi \\
0, \text { otherwise }
\end{array}\right.
$$

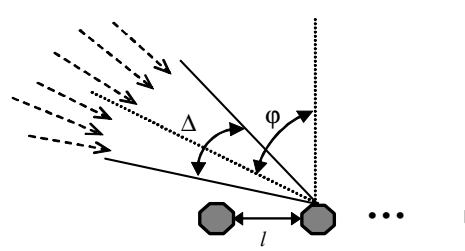

Fig 1. Incoming multipath signals arrive to the linear antenna array within $\pm \Delta / 2$ of mean angle $\varphi$

The normalized correlation between two omni-directional antennas can be expressed as

$$
R(l)=\int_{-\pi}^{\pi} f_{\theta}(\theta) \exp [j z \sin \theta] d \theta
$$

where $z=2 \pi l / \lambda, l$ is the element spacing, $\lambda$ is the wavelength, and $j$ is the imaginary unit. A simple but still accurate approximation of the Bessel series expansion for small $\Delta<<1$ can be derived using $\sin \beta \approx \beta$ (valid for small $\beta$ ), and performing integration in (2) $[2,6,9]$ 


$$
R_{u}(l) \approx \exp (j z \sin \varphi) \sin (\Delta z \cos \varphi / 2)
$$

where $\operatorname{sinc}(x)=\sin (x) /(x)$.

Measurements indicate that multipath power density is not uniform distributed. It can be modeled as truncated Gaussian, truncated Laplacian or other distribution. Furthermore, [8] demonstrates that the Laplacian distribution gives better match than uniform or truncated Gaussian one in both urban and rural areas. For the truncated Gaussian distribution, the pdf is

$$
f_{\theta}(\theta)=\frac{Q_{g}}{\sqrt{2 \pi} \sigma_{g}} e^{-\frac{(\theta-\varphi)^{2}}{2 \sigma_{g}}} \quad-\pi+\varphi \leq \theta \leq \pi+\varphi
$$

where $Q_{g}=1 / \operatorname{erf}\left(\gamma_{g}\right), \gamma_{g}=\pi /\left(\sqrt{2} \sigma_{g}\right)$, and the spatial correlation can be approximated as [6]

$$
R_{g}(l) \approx \exp \left(j z \sin \varphi-\left(\sigma_{g} z \cos \varphi\right)^{2} / 2\right)
$$

For the truncated Laplacian distribution, the pdf is

$$
f_{\theta}(\theta)=\frac{Q_{l}}{\sqrt{2} \sigma_{l}} e^{-\frac{\sqrt{2}|\theta-\varphi|}{\sigma_{l}}} \quad-\pi+\varphi \leq \theta \leq \pi+\varphi
$$

where $Q_{L}=1 /\left[1-\exp \left(-\gamma_{l}\right)\right]$ and $\gamma_{l}=\sqrt{2} \pi / \sigma_{l}$, and the spatial correlation is approximately

$$
R_{l}(l) \approx \exp (j z \sin \varphi) /\left[1+\left(\sigma_{l} z \cos \varphi\right)^{2} / 2\right]
$$

For the small variance, $\sigma_{g} \ll 1, \sigma_{l} \ll 1$, (3), (5) and (7) have similar expansion terms [6] [10] by setting the same angular spread for these distributions, i.e. $\sigma=\sigma_{g}=\sigma_{l}=\Delta / 2 \sqrt{3}$,

$$
\begin{aligned}
& R_{g}(l) \approx\left[1-\frac{1}{2}(\sigma z \cos \varphi)^{2}+\frac{1}{8}(\sigma z \cos \varphi)^{4}\right] e^{j z \sin \varphi} \\
& R_{u}(l) \approx\left[1-\frac{1}{2}(\sigma z \cos \varphi)^{2}+\frac{3}{40}(\sigma z \cos \varphi)^{4}\right] e^{j z \sin \varphi} \\
& R_{l}(l) \approx\left[1-\frac{1}{2}(\sigma z \cos \varphi)^{2}+\frac{1}{4}(\sigma z \cos \varphi)^{4}\right] \mathrm{e}^{j z \sin \varphi}
\end{aligned}
$$

These approximations are correct provided that $\sigma z|\cos \varphi|<1$. Comparing (8), (9) and (10), one can conclude that the correlation coefficient is very similar provided the average angle $\varphi$ is not too small (not too close to the end-fire direction), and the antenna spacing is not too large.

\section{Single-Cluster Channel Model IN $u$ - DOMAIN}

The spatial correlation can be alternatively derived using the angular spectrum of the incoming multipath. The spatial correlation and the angular spectrum of the effective sources are related through the Fourier transform [11],

$$
P_{u}(u)=\frac{1}{2 \pi} \int_{-\infty}^{\infty} R(l) e^{-j u l} d l, R(l)=\int_{-\infty}^{\infty} P_{u}(u) e^{j u l} d u
$$

where $u=k \sin \theta=2 \pi \sin \theta / \lambda$ is spatial frequency. We further use the following notations,

$$
P_{u}(u)=F T[R(l)], \quad R(l)=\operatorname{IFT}\left[P_{u}(u)\right]
$$

The power density of the effective scatterer distribution in the $u$-domain is expressed as $P_{u}(u)=P_{\theta}(\theta) / \sqrt{k^{2}-u^{2}}$, where $P_{\theta}(\theta)=f_{\theta}(\theta) g^{2}(\theta)$ is the power of the effective scatterer distribution in the angular $(\theta)$ domain, $g(\theta)$ is the antenna pattern. In this paper, we consider only omni directional antennas $(g(\theta)=1)$. Defining $\beta \triangleq \theta-\varphi$, $u_{0} \triangleq k \sin \varphi$, where $\varphi$ is the mean value of $\theta$, one obtains $f_{\theta}(\theta)=f_{\beta}(\theta-\varphi), \quad P_{\theta}(\theta)=f_{\beta}(\theta-\varphi) ; f_{\beta}(\beta)$ is the angular pdf centered about the broadside direction. For small $\beta, \sqrt{k^{2}-u^{2}} \approx \sqrt{k^{2}-u_{0}^{2}}=k \cos \varphi$. Using the Taylor series expansion,

$$
\theta=\sin ^{-1} \frac{u}{k} \approx \sin ^{-1} \frac{u_{0}}{k}+\frac{u-u_{0}}{\sqrt{k^{2}-u^{2}}},
$$

and $\beta \approx\left(u-u_{0}\right) /(k \cos \varphi)$. Using this,

$$
P_{u}(u) \approx \frac{1}{k \cos \varphi} f\left(\frac{u-u_{0}}{k \cos \varphi}\right)
$$

Combining (12) and (11), one obtains the spatial correlation,

$$
R(l)=I F T\left[\frac{1}{k \cos \varphi} f\left(\frac{u-u_{0}}{k \cos \varphi}\right)\right]
$$

Using the scaling and shifting properties of Fourier transform, (13) can be further simplified as

$$
R(l)=e^{j u_{o} l} F(k \cos \varphi \cdot l)
$$

where $F(x)=\operatorname{IFT}[f(\beta)]$ is the correlation of the angular pdf centered about the broadside direction: if $\varphi=0$, $R_{0}(l)=F(k l)$. Non-zero $\varphi$ results in the scale factor $e^{j u_{0} l}$ in the correlation coefficient and the scale factor $\cos \varphi$ in the function argument. Using (14), one can easily obtain the results for uniform, Gaussian and Laplacian pdfs, i.e. (3), (5) and (7). Note, however, that (14) is valid for any angular pdf with small angular spread.

Using the moment property of Fourier transform and the fact that the odd order moments of $f(\beta)$ are zero (because $f(\beta)=f(-\beta))$, one obtains for $i=0,1,2, \ldots$,

$$
S_{2 i+1}=\int_{-\infty}^{\infty} \beta^{2 i+1} f(\beta) d \beta=\left.\frac{d^{2 i+1} F(x)}{d x^{2 i+1}}\right|_{x=0}=0
$$

The even order moments of $f(\beta)$ are

$$
S_{2 i}=\int_{-\infty}^{\infty} \beta^{2 i} f(\beta) d \beta=\left.(-1)^{i} \frac{d^{2 i} F(x)}{d x^{2 i}}\right|_{x=0}
$$

In particular, 


$$
S_{2}=\int_{-\infty}^{\infty} \beta^{2} f(\beta) d \beta=-\left.\frac{d^{2} F}{d x^{2}}\right|_{x=0}=\sigma^{2}
$$

The Taylor series expansion of $F(x)$,

$$
F(x)=F(0)+\left.\sum_{n=1}^{\infty} \frac{x^{n}}{n !} \frac{d^{n} F}{d x^{n}}\right|_{x=0}
$$

can be expressed as

$$
F(x)=1-\frac{x^{2}}{2} \sigma^{2}+\frac{x^{4}}{4 !} S_{4}-\frac{x^{6}}{6 !} S_{6}+\cdots,
$$

Using (14), generic spatial correlation is

$$
R(l)=e^{j u_{o} l}\left(1-\frac{(k l \cos \varphi)^{2}}{2} \sigma^{2}+\frac{(k l \cos \varphi)^{4}}{4 !} S_{4}+\cdots\right)
$$

This equation immediately explains the results (8) - (10) for the uniform, truncated Gaussian and truncated Laplacian distributions.

One can use (14) and (18) to derive the compact expressions and approximations for various angular pdfs. As an example, using the triangle pdf,

$$
f(\beta)=\frac{\Lambda\left(\beta / \sigma_{t}\right)}{\sigma_{t}}, \Lambda(t)=\left\{\begin{array}{lr}
t+1 & -1 \leq t<0 \\
-t+1 & 0 \leq t<1
\end{array},\right.
$$

$F(x)=\operatorname{IFT}(f(\beta))=\operatorname{sinc}^{2}\left(\sigma_{t} x / 2\right)$, and the compact form of the spatial correlation is

$$
R(l)=e^{j k \sin \varphi \cdot l} \operatorname{sinc}^{2}\left(\sigma_{t} k \cos \varphi \cdot l / 2\right)
$$

The second moment of of $f(\beta)$ is $S_{2}=\sigma_{t}^{2} / 6$, and the forth moment of of $f(\beta)$ is $S_{4}=\sigma_{t}^{4} / 15$. Setting $\sigma_{t}=\sqrt{6} \sigma$ to achieve the same angular spread with the uniform, Laplacian and Gaussian pdfs, and using (18), one obtains

$$
R(l)=e^{j u_{o} l}\left(1-\frac{1}{2}(k l \sigma \cos \varphi)^{2}+\frac{1}{10}(k l \sigma \cos \varphi)^{4}+\cdots\right)
$$

Comparing (19) with (8)-(10), and noticing that $k l=z$, $u_{o} l=z \sin \varphi$, one can observe that the magnitude of correlation with the triangle pdf is between those with the uniform and Gaussian pdf (see Fig 3).

Asymptotically, $\quad R(l) \approx 0$ if $k \sigma l \cos \varphi$ is large enough, and $R(l)=1$ for $k \sigma l \cos \varphi=0$ for any pdf model. On the other hand, the magnitude of correlation decreases when $k \sigma l \cos \varphi$ increases. If $k l \sigma|\cos \varphi|>1.1,|R(l)|<0.5$, and the system performance is not affected significantly by the correlation (see [6] and Fig 4). For $k l \sigma|\cos \varphi|<1.1$, one can approximate (18) as

$$
R(l)=e^{j u_{o} l}\left(1-(k l \sigma \cos \varphi)^{2} / 2\right)
$$

If $k \sigma l \cos \varphi$ is fixed, (20) shows that any pdf model will produce roughly the same spatial correlation. Overall, the performance predictions based on different models will be roughly the same in terms of correlation. We conclude that the assumption of uniform angular pdf does not limit significantly the accuracy of the single cluster angular model. Since uniform distribution is much easier to work with, it should be used for the system performance prediction.

\section{TWO-Cluster MOdEL}

Measurements indicated that multipath arrives frequently in more than one cluster [3][4][5]. The single cluster channel model has been generalized to multiple clusters [6][7][9]. In particular, [7] derived a generic expression for correlation coefficient using Bessel series. However, in the case of two clusters with uniform pdf, it is especially simple[9]. We assume the angular pdf of the form,

$$
p(\varphi)=\left\{\begin{array}{l}
\frac{1}{\Delta_{1}+\Delta_{2}},\left\{\begin{array}{l}
-\Delta_{1} / 2+\varphi_{1} \leq \varphi \leq \Delta_{1} / 2+\varphi_{1}, \\
-\Delta_{2} / 2+\varphi_{2} \leq \varphi \leq \Delta_{2} / 2+\varphi_{2}
\end{array}\right. \\
0, \text { elsewhere }
\end{array}\right.
$$

and only study two symmetric clusters in this paper. Hence, $\Delta_{1}=\Delta_{2}=\Delta$ and $\varphi_{1}=-\varphi_{2}=\varphi$. The scenario is illustrated in Fig. 2 .

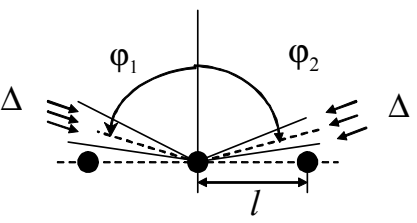

Fig 2. Incoming multipath is concentrated in two clusters.

As demonstrated in [10], the compact approximation for the spatial correlation is

$$
R_{2}(l) \approx \cos (z \sin \varphi) \operatorname{sinc}(\Delta z \cos \varphi / 2)
$$

This approximation agrees well with the rigorous results and Monte-Carlo simulations. Although the real parts of (3) and (22) are the same, their magnitudes are very different. In fact, the single-cluster magnitude correlation is the envelope of the two-cluster correlation and the cosine function in (22) results in oscillation in the latter case.

Now let's consider this model in the u-domain. Using the Fourier transform properties, (14) can be generalized to the two cluster case as follows

$$
F T\left[R_{2}(l)\right]=\frac{1}{2 k \cos \varphi}\left[f\left(\frac{u-u_{0}}{k \cos \varphi}\right)+f\left(\frac{u+u_{0}}{k \cos \varphi}\right)\right]
$$

where $R_{2}(l)=R(l) \cdot e^{-j u_{0} l} \cdot \cos \left(u_{0} l\right)$. The RHS of (23) is the angular spectrum of the two-cluster channel, and $R_{2}(l)$ is the spatial correlation, which can be related to the singlecluster one,

$$
R_{2}(l)=\cos \left(u_{0} l\right) \cdot F(k \cos \varphi \cdot l)
$$


For instance, for the uniform pdf, $F(x)=\operatorname{sinc}(x \Delta / 2)$, which can be written as

$$
R_{2}(l)=\cos (z \sin \varphi) \operatorname{sinc}(\Delta z \cos \varphi / 2)
$$

This result agrees with (22), which was derived from the angular pdf directly without using Fourier transform [10].

Fig 3 compares correlations predicted by the different pdf models considered above. Clearly, the uniform and Laplacian distributions give the most pessimistic and most optimistic predictions, respectively, which is in the agreement with the arguments in single cluster channel. The triangle distribution is between uniform and Gaussian pdf

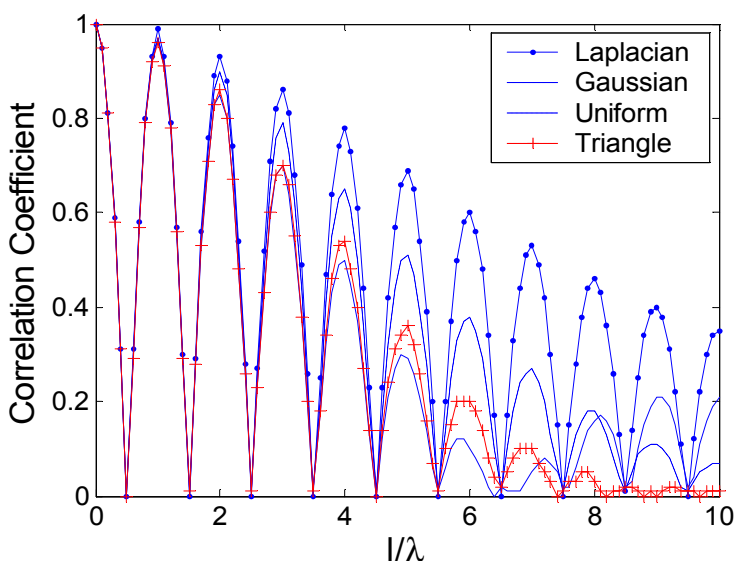

Fig 3. Correlation coefficient versus element spacing for the case of two symmetric clusters. $\Delta=10^{0}, \varphi=30^{\circ}$ with uniform, triangle, Gaussian and Laplacian pdf

\section{MIMO CAPACITY}

Using the approach of [2] and the correlation models above, the MIMO capacity can be evaluated. As an example, we consider a MIMO system of $n_{T}=n_{R}=10$ with $\mathrm{SNR}=20$ $\mathrm{dB}$ per receive antenna, and a two-cluster channel with 20 multipath signals arriving at each cluster with angular spread $\sigma_{2}=\sigma_{1}=5^{0} / \sqrt{3}, \varphi_{1}=-30^{0} \quad, \quad \varphi_{2}=30^{\circ} \quad$ (i.e., symmetric). The transmit array elements are assumed to be uncorrelated - all the correlation is concentrated at the $\mathrm{Rx}$ end. The mean capacity of the channel is shown in Fig 4. Clearly, given the same parameters (i.e. angular spread and the mean AOA), all the different pdfs result in almost the same MIMO capacity. Comparison with the correlation curve indicates that the peaks of correlation correspond to notches of the capacity and vise versa. Also, the capacity oscillations, which do not appear in the single-cluster model [2], are apparent.

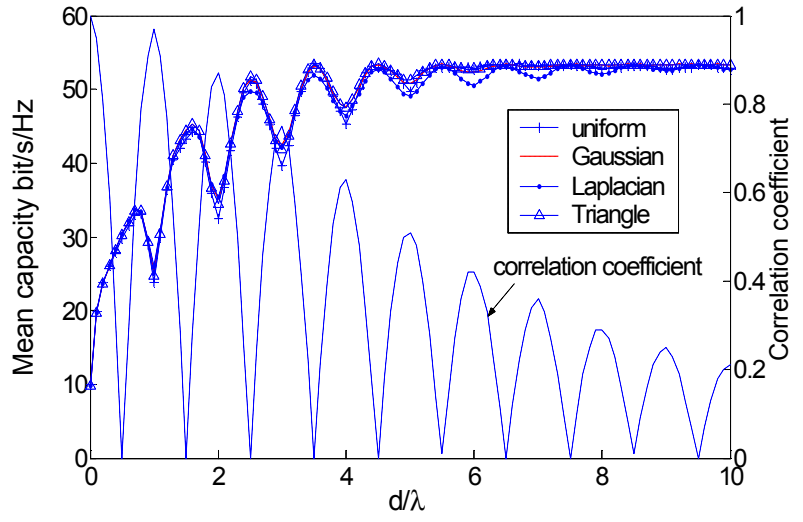

Fig 4. 10x10 MIMO channel capacity of a two-symmetric-cluster channel for various multipath AOA pdf

\section{References}

[1] J. Salz, J.H. Winters, "Effect of Fading Correlation on Adaptive Arrays in Digital Mobile Radio," IEEE Trans. Veh. Tech., V.43, N.4, pp. 1049-1057,Nov. 1994

[2] S. Loyka, G. Tsoulos, "Estimating MIMO System Performance Using the Correlation Matrix Approach," IEEE Commun. Lett., V. 6, N.1,pp. 19-21, Jan. 2002

[3] A. A. Saleh and R. A. Valenzuela, "A statistical model for indoor multipath propagation," IEEE JSAC, V. SAC-5, pp. 128-137, Feb. 1987

[4] Q. Spencer, M. Rice, B. Jeffs, and M. Jensen, "A statistical model for angle of arrival in indoor multipath propagation," in Proc. 1997 IEEE VTC, V. 3, Phoenix, AZ, pp. 1415-1419, May 1997

[5] K. I. Pedersen, P. E. Mogensen and B. H. Fleury, “A stochastic model of the temporal and azimuthal dispersion seen at the base station in outdoor propagation environments," IEEE Trans. Veh. Tech., V. 49, N.2, pp.437447 , Mar. 2000,

[6] R. M. Buehrer, "The Impact of Angular Energy Distribution on Spatial Correlation," IEEE VTC, Vancouver, Canada, Sep. 2002, pp.1173-1177

[7] L. Schumache et al, "From Antenna Spacing to Theoretical Capacities - Guidelines for Simulating MIMO Systems," PIMRC, Lisbon, Portugal, Sep. 2002, pp. 587-592.

[8] Q.H. Spencer, B.D. Jeffs, M.A. Jensen, and A.L. Swindlehurst, "Modeling the Statistical Time and Angle of Arrival Characteristics of an Indoor Multipath Channel," IEEE JSAC., V. 18, N. 3, Mar. 2000, pp. 347-360

[9] G. Zhao, S. Loyka, " Impact of Multipath Clustering on the Performance of MIMO System", IEEE WCNC 2004, Atlanta, Georgia, USA, Mar. 2004

[10] G. Zhao, S. Loyka, " Performance Study of MIMO System in a Clustered Multipath Channel", IEEE CCECE , Niagara Falls, Canada, May. 2004

[11] R.Vaughan, "Spaced directive antennas for mobile communications by the Fourier transform method," IEEE Trans. on Antennas and Propagation, V. 48, N. 7, pp. $1025-$ 1032, July 2000 